MATHEMATICS OF COMPUTATION

Volume 81, Number 277, January 2012, Pages 569-583

S $0025-5718(2011) 02502-0$

Article electronically published on May 13, 2011

\title{
ARCHIMEDEAN MAPS OF HIGHER GENERA
}

\author{
JÁN KARABÁŠ AND ROMAN NEDELA
}

\begin{abstract}
The paper focuses on the classification of vertex-transitive polyhedral maps of genus from 2 to 4 . These maps naturally generalise the spherical maps associated with the classical Archimedean solids. Our analysis is based on the fact that each Archimedean map on an orientable surface projects onto a one- or a two-vertex quotient map. For a given genus $g \geq 2$ the number of quotients to consider is bounded by a function of $g$. All Archimedean maps of genus $g$ can be reconstructed from these quotients as regular covers with covering transformation group isomorphic to a group $\mathrm{G}$ from a set of $g$-admissible groups. Since the lists of groups acting on surfaces of genus 2,3 and 4 are known, the problem can be solved by a computer-aided case-to-case analysis.
\end{abstract}

\section{INTRODUCTION}

By a map $\mathbf{M}$, we mean a 2-cell decomposition of a compact connected orientable surface $\mathcal{S}_{g}$ of genus $g$. In other words, a map $\mathbf{M}$ can be described as a 2-cell embedding $\varepsilon: \Gamma \hookrightarrow \mathcal{S}_{g}$ of the underlying graph $\Gamma$ into a surface $\mathcal{S}_{g}$. The connected components of $\mathcal{S}_{g} \backslash \varepsilon(\Gamma)$ are called faces. Given a map M, an automorphism of the underlying graph $\Gamma$ which extends to a self-homeomorphism of $\mathcal{S}_{g}$ is called an automorphism of $\mathbf{M}$. A map $\mathbf{M}$ is vertex-transitive if its automorphism group Aut(M) acts transitively on vertices of $\Gamma$. In a vertex-transitive map on an orientable surface, the group of orientation-preserving automorphisms $\mathrm{Aut}^{+}(\mathbf{M})$ acts on the vertex-set either with one or with two orbits.

Graphs considered in this paper may have loops, multiple edges and semiedges. More precisely, a graph is a quadruple $\Gamma=(D, V ; I, L)$, where $D=D(\Gamma)$ and $V=V(\Gamma)$ are disjoint nonempty finite sets, $I: D \rightarrow V$ is a surjective mapping and $L$ is an involutory permutation on $D$. The elements of $D$ and $V$ are darts and vertices, respectively, $I$ is the incidence function assigning to every dart its initial vertex and $L$ is the dart-reversing involution. The orbits of the group $\langle L\rangle$ on $D$ are edges of $\Gamma$. It may happen that $x . L=x$ for some dart $x \in \Gamma$, and in this case the corresponding edge is called a semiedge. If $I(x . L)=I(x)$ but $x . L \neq x$, then the corresponding edge is called a loop. The remaining edges are called links. Two links $\{x, x . L\}$ and $\{y, y . L\}$ are parallel if $I(x)=I(y)$ and $I(x . L)=I(y . L)$, or $I(x)=I(y . L)$ and $I(x . L)=I(y)$. A graph without semiedges, loops and parallel

Received by the editor September 14, 2007 and, in revised form November 4, 2010.

2010 Mathematics Subject Classification. Primary 05C30; Secondary 05C10, 05C25.

Key words and phrases. Polyhedron, Archimedean solid, map, surface, group, graph embedding.

Both authors were partially supported by the grants APVV-51-009605 and VEGA 1/0722/08, grants of Slovak Ministry of Education.

(C)2011 American Mathematical Society Reverts to public domain 28 years from publication 
links is called a simple graph. The above definition of a graph follows the approach of Jones and Singermann [17] and is the same as in [25].

A (convex) polyhedron is defined in [15, 16] as the convex hull of a finite set of points in the Euclidean space. By Steinitz's theorem [30] a graph forms a 1-skeleton of a convex polyhedron if and only if it is planar and 3-connected. Therefore a simple graph is called polyhedral if and only if it is planar and 3-connected. A 2-cell decomposition of the sphere given by a 2-cell embedding of a polyhedral graph into the sphere will be called a spherical polyhedral map. Note that a 2-cell embedding of a polyhedral graph into the sphere is uniquely determined by the graph, hence there is a correspondence between polyhedral graphs, polyhedral maps and polyhedra. To generalise the concept of a polyhedral map to higher genera we shall relax the condition on polyhedrality as follows.

We say that two faces of a map are adjacent if they are incident to the same vertex. In a spherical polyhedral map a boundary of a face is a simple cycle and the boundary cycles of two adjacent faces intersect either in a single vertex, or in a single edge together with the two incident vertices. Following Mohar and Thomassen [24, Proposition 5.5.12] and Brehm and Schulte [4], we say that a map on a surface of genus $g$ is polyhedral of genus $g$ if the boundary of every face is a simple cycle and the boundary cycles of any two faces are either disjoint or intersect either in a single vertex or in a single edge (together with the two incident vertices). By Mohar and Thomassen [24, Proposition 5.5.12] the underlying graph of a polyhedral map is simple and 3-connected. An Archimedean map is a polyhedral map such that its automorphism group is transitive on its vertices. Note that in [26] a weaker definition of polyhedrality is used.

The classification of classical Archimedean solids (maps) is done by their local types. A local type of a map $\mathbf{M}$ at a vertex $v$ is nothing but a cyclic sequence of lengths of faces incident to $v$ following a given global orientation of the surface. In the case of Archimedean maps the local types do not depend on a choice of a vertex. Therefore for an Archimedean map $\mathbf{M}$ we can talk about a type of $\mathbf{M}$. We write the type of a map $\mathbf{M}$ in a multiplicative form (e.g., (3.4.4.6.4) $=\left(3.4^{2} .6 .4\right)$ or $\left.(3.3 .3 .3)=\left(3^{4}\right)\right)$ also known as Cundy and Rollett symbol [9]. Since we shall consider a map and its mirror image to be isomorphic, a local type and its mirror image will be considered to be the same. As a rule we shall use the lexically minimal representative of local type of a map. For each local type there exists a universal Archimedean map (tiling) of the sphere, Euclidean or hyperbolic plane covering every Archimedean map of that type; see 8 .

There are 13 classical Archimedean solids (15 if the mirror images of two enantiomorphs are counted separately). Thus there are 13 (15) associated spherical Archimedean maps. Moreover, the five Platonic solids give rise to another five spherical Archimedean maps. To complete the list of spherical Archimedean maps we include the infinite families of maps associated with the prisms and anti-prisms. For more information see e.g. the Wikipedia 32 .

Toroidal Archimedean maps are quotients of the uniform (vertex-transitive) tilings of the Euclidean plane [16, p. 63]. There are 11 distinct Archimedean tilings of the Euclidean plane $E_{2}$ (12 if the mirror images of two enantiomorphs of type $\left(3^{4} .6\right)$ are counted separately). Each toroidal Archimedean map can be constructed as a quotient of one of the universal tilings by a group of translations. Each of the universal tilings give rise to an infinite family of toroidal Archimedean maps. A more 
detailed classification can be found in [31. For further information see [1, 26, 27, 31] and [14, pp. 291-295].

From the above notes it is clear that there are infinitely many Archimedean maps of both genus 0 and genus 1 . In contrast, by the well-known Riemann-Hurwitz bound combined with Proposition 5 , there are finitely many Archimedean maps for each genus $g, g \geq 2$. In particular, we have the following proposition.

Proposition 1 (Riemann-Hurwitz bound, [13). Given a vertex-transitive map $\mathbf{M}$ of genus $g>1$ the order of $\operatorname{Aut}(\mathbf{M})$ is bounded by $|\operatorname{Aut}(\mathbf{M})| \leq 168(g-1)$ and the order of the group of orientation-preserving automorphisms is bounded by $\mid$ Aut $^{+}(\mathbf{M}) \mid \leq 84(g-1)$. In particular, the number of vertices of $\mathbf{M}$ is at most $168(g-1)$.

In the present paper the following classification problem will be considered.

Problem. Classify isomorphism classes of Archimedean maps of given genus $g>1$. A similar problem, the classification of regular maps of genus $2 \leq g \leq 101$, has already been solved by Conder [7. The main result of our paper written in the enumerative form follows. Complete lists of Archimedean maps of genus 2, 3, 4 can be found at the website [18.

Theorem 2 (Main Theorem). There are 17, 103 and 111 isomorphism classes of Archimedean maps of genus 2, 3, 4, respectively.

\section{TWO-DIMENSIONAL ORBIFOLDS AND MAPS ON ORBIFOLDS}

The idea of an orbifold comes from geometry of manifolds, where they are defined in a more general setting. Generally, orbifolds can be viewed as homomorphic images of manifolds, in particular, a quotient orbifold is induced by an action of a discrete group of automorphisms of a manifold.

For the purpose of this paper we define an (orientable) orbifold to be an orientable surface of genus $\gamma \geq 0$ together with a distinguished finite set of points $\mathcal{B}=\left\{b_{1}, b_{2}, \ldots, b_{r}\right\}$, to each point $b_{i}$ in $\mathcal{B}$ there is associated an integer $m_{i} \geq 2$, $i=1,2, \ldots r$. The numbers $m_{1}, m_{2}, \ldots, m_{r}$ are called branch-indices. Each orbifold is determined by its signature $\left(\gamma ; m_{1}, m_{2}, \ldots, m_{r}\right)$, where the branch-indices are ordered in a nondecreasing sequence $m_{1} \leq m_{2} \leq \cdots \leq m_{r}$.

Every vertex-transitive map $\mathbf{M}$ on a surface of genus $g$ covers regularly a quotient map $\overline{\mathbf{M}}=\mathbf{M} / \operatorname{Aut}^{+}(\mathbf{M})$. The quotient map $\overline{\mathbf{M}}$ is of genus $\gamma \leq g$. The underlying surface $S_{\gamma}$ can be viewed as a two-dimensional quotient orbifold $\mathcal{O}\left(\gamma ; m_{1}, m_{2}, \ldots, m_{r}\right)$ $=S_{g} / \operatorname{Aut}^{+}(\mathbf{M})$, where the parameters $\gamma, g, m_{1}, \ldots, m_{r}$ are related by the RiemannHurwitz equation:

$$
\begin{gathered}
2-2 g=\left|\operatorname{Aut}^{+}(\mathbf{M})\right|\left(2-2 \gamma-\sum_{i=1}^{r}\left(1-\frac{1}{m_{i}}\right)\right), m_{i} \geq 2, \\
\left|\operatorname{Aut}^{+}(\mathbf{M})\right| \equiv 0 \bmod m_{i} .
\end{gathered}
$$

Note that given a surface, its admissible quotient orbifolds were classified by Broughton [5] for genus 2 and 3, and by Bogopolski [2] for genus four. Lists of admissible orbifolds for surfaces of higher genera can be found in [19.

Our approach is based on the fact that given a quotient orbifold $\mathcal{O}$ we can identify all the potential quotient maps $\overline{\mathbf{M}}=\mathbf{M} / \operatorname{Aut}^{+}(\mathbf{M})$ on $\mathcal{O}$ which have by definition one or two vertices. Moreover, given a map $\overline{\mathbf{M}}$ on $\mathcal{O}$, we can reconstruct the covering map $\mathbf{M}$ over $\overline{\mathbf{M}}$ and verify whether it is polyhedral or not. To do this 
effectively we first need a combinatorial description of a map on an orbifold and to derive more facts on the quotients of Archimedean maps. The reconstruction is explained in the following section.

It is well known [17, 25] that a map on an orientable surface can be described by means of a triple $\mathbf{M}=(D ; R, L)$, where $D$ is a set of darts of $\mathbf{M}, R, L \in \operatorname{Sym}(D)$ are permutations of darts of $\mathbf{M}$ such that $L^{2}=1$ and $\langle R, L\rangle$ is transitive on $D$. The permutation $R$ is called the rotation of $\mathbf{M}$ and $L$ is called the dart reversing involution. The orbits of $R$ are identified with the set $V$ of vertices of $\mathbf{M}$, the orbits of $L$ are identified with the set $E$ of edges of $\mathbf{M}$ and the cycles of $R L$ form boundary walks of the set $F$ of faces of $\mathbf{M}$. The edges that correspond to orbits of length one are called semiedges. A valency val $(x)$ of a vertex, edge or face $x$ is the size of the corresponding orbit, i.e., $\operatorname{val}(x)=|x|$. It follows that the valency of an edge $e$ is either one or two depending on whether $e$ is a semiedge or not.

Let $\mathbf{M}_{1}=\left(D_{1} ; R_{1}, L_{1}\right)$ and $\mathbf{M}_{2}=\left(D_{2} ; R_{2}, L_{2}\right)$. Then a map homomorphism $\varphi: \mathbf{M}_{1} \rightarrow \mathbf{M}_{2}$ is a mapping $\varphi: D_{1} \rightarrow D_{2}$ such that $\varphi R_{1}=R_{2} \varphi$ and $\varphi L_{1}=L_{2} \varphi$. By transitivity of $\left\langle R_{2}, L_{2}\right\rangle$ every map homomorphism is surjective, therefore map homomorphisms are called coverings. The orientation preserving map automorphisms are permutations of $D$ commuting with both $R$ and $L$. By transitivity of $\langle R, L\rangle$ the action of $\mathrm{Aut}^{+}(\mathbf{M})$ on $D$ is semiregular (see Dixon and Mortimer [10, Theorem 4.2a]). A permutation $\tau$ of $\mathbf{M}$ is an orientation reversing automorphism of $\mathbf{M}$ if $\tau R \tau^{-1}=R^{-1}$ and $\tau L \tau=L$. The automorphism group Aut(M) is formed by orientation preserving and orientation reversing automorphisms of $\mathbf{M}$. Since a composition of two orientation reversing automorphisms belongs to $\mathrm{Aut}^{+}(\mathbf{M})$, we have $\left[\operatorname{Aut}(\mathbf{M}): \operatorname{Aut}^{+}(\mathbf{M})\right] \leq 2$. If there are no orientation reversing automorphisms $\mathbf{M}$ is called chiral, otherwise it is called reflexible. An orientation reversing automorphism of order two fixing a vertex, an edge or a face is called a reflection.

Every vertex-transitive map $\mathbf{M}$ with a group of orientation-preserving automorphisms $\mathrm{G} \leq \operatorname{Aut}^{+}(\mathbf{M})$ covers a quotient $\overline{\mathbf{M}}=\mathbf{M} / \mathbf{G}=(\bar{D} ; \bar{R}, \bar{L})$ of genus $\gamma$, where $\bar{D}=\left\{[x]_{\mathrm{G}} ; x \in D\right\},[x] \bar{R}=[x R]$ and $[x] \bar{L}=[x L]$. The mapping $x \mapsto[x]_{\mathrm{G}}$ is a covering $\mathbf{M} \rightarrow \overline{\mathbf{M}}$ called a standard covering.

Proposition 3. Let $\mathbf{M}$ be an Archimedean map on an orientable surface. Then the quotient map $\overline{\mathbf{M}}=\mathbf{M} / \mathrm{Aut}^{+}(\mathbf{M})$ is either a one-vertex map, or a two-vertex map.

Proof. Since $\left[\operatorname{Aut}(\mathbf{M}): \operatorname{Aut}^{+}(\mathbf{M})\right] \leq 2$, the group of orientation preserving automorphisms acts on the vertex set either with one, or with two orbits. It follows that the quotient map has at most two vertices.

It follows that Archimedean maps are of two kinds, which will be treated separately. If $\mathrm{Aut}^{+}(\mathbf{M})$ acts on the vertices of $\mathbf{M}$ inducing one orbit, then $\mathbf{M}$ will be called an Archimedean map of type I. In the other case if $\mathrm{Aut}^{+}(\mathbf{M})$ acts on the vertices of $\mathbf{M}$ inducing two orbits, $\mathbf{M}$ will be called an Archimedean map of type II.

The quotient map $\overline{\mathbf{M}}=\mathbf{M} / \mathrm{Aut}^{+}(\mathbf{M})$ lies on a surface of genus $\gamma \leq g$. The genus $\gamma$ can be counted by the Euler-Poincaré formula $2-2 \gamma=\bar{V}-\bar{E}+\bar{F}$. Moreover, the covering $f: \mathbf{M} \rightarrow \overline{\mathbf{M}}$ associates with each vertex, edge, or face $\bar{x}=f(x)$ an integer $b(\bar{x})=\frac{\operatorname{val}(x)}{\operatorname{val}(f(x))}$. It follows that $f$ determines an orbifold $\mathcal{O}$ with signature $\left(\gamma ; m_{1}, m_{2}, \ldots, m_{r}\right)$, where the set of branch-indices is given by $\{b(\bar{x}) \mid b(\bar{x}) \geq 2$ and $x \in V \cup E \cup F\}$. In general, a map $\mathbf{M}=(D ; R, L)$ is on an 
orbifold $\mathcal{O}\left(\gamma ; m_{1}, m_{2}, \ldots, m_{r}\right)$ if it is of genus $\gamma$ and a function $b: V \cup E \cup F \rightarrow$ $\left\{1, m_{1}, m_{2}, \ldots, m_{r}\right\}$ satisfying the following conditions is given:

(M1) Let $x$ be an edge. Then $b(x) \leq 2$, and $b(x)=2$ if and only if $x$ is a semiedge.

(M2) For each $i \in\{1,2, \ldots, r\}$ there is exactly one $x \in V \cup E \cup F$ such that $b(x)=m_{i}$.

If $b$ is defined by setting $b(\bar{x})=\frac{\operatorname{val}(x)}{\operatorname{val}(f(x))}$, for some regular covering $f: \mathbf{M} \rightarrow \overline{\mathbf{M}}$, we say that $b$ is induced by $f$. We will say that a covering $f: \mathbf{M} \rightarrow \overline{\mathbf{M}}$ is regular if the group of covering transformations acts transitively (and hence regularly) on each fibre over a dart. For more details on regular coverings between maps see [22].

The above definition of a map on an orbifold comes from [23]. The following proposition is a useful extension of Proposition 3 .

Proposition 4. Let $\mathbf{M}$ be an Archimedean map of type II. Then an orientation reversing automorphism $\tau$ of $\mathbf{M}$ transposing the two vertex orbits projects into orientation reversing automorphism $\bar{\tau}$ of $\overline{\mathbf{M}}$.

In particular, the branch-indices of a face $\bar{F}$ and its image $\bar{\tau}(\bar{F})$ coincide and the branch-indices of the two vertices are the same.

Proof. The following diagram has to commute:

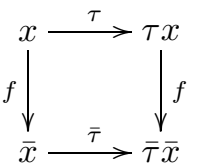

Let $G=\operatorname{Aut}^{+}(\mathbf{M})$. Set $f: \mathbf{M} \rightarrow \overline{\mathbf{M}}, f: x \mapsto[x]_{G}$, where $[x]_{G}$ denotes the orbit of $G$ containing a dart $x$ of $\mathbf{M}$. Since $G \unlhd \operatorname{Aut}(\mathbf{M})$,

$$
[g \tau x]_{G}=\left[\tau g^{\prime} x\right]_{G}=[\tau x]_{G} .
$$

It follows that $\bar{\tau}:[x]_{G} \mapsto[\tau x]_{G}$ is well defined. Moreover, valencies of $\bar{F}$ and $\bar{\tau}(\bar{F})$, as well as the valencies of their preimages in $\mathbf{M}$, are the same. The result follows.

For technical reasons we slightly modify a notation of an orbifold, where a oneor two-vertex map is embedded. Let $\overline{\mathbf{M}}$ be a one-vertex map on an orbifold $\mathcal{O}$. Let $n$ be the sum of the numbers of its faces and semiedges. For each face and for each semiedge let $m_{i} \geq 1, i=1, \ldots, n$, denote the respective branch-index, and let $m_{0} \geq 1$ be a branch-index associated with the unique vertex of $\overline{\mathbf{M}}$. In other words, we admit that some branch-indices are equal to one. With this notation in mind we write $\mathcal{O}=\mathcal{O}\left(\gamma ; m_{0}, m_{1}, m_{2}, \ldots, m_{n}\right), m_{i} \geq 1$ for $i=0,1, \ldots, n$, with the convention that $m_{0} \geq 1$ is a branch-index of the unique vertex. Alternatively, if $\mathbf{M}$ is a 2-vertex quotient map, the respective orbifold will be described as $\mathcal{O}=\left(\gamma ; m_{0}^{2}, m_{1}, \ldots, m_{n}\right)$ with the convention that the two branch-points of index $m_{0} \geq 1$ are associated with the two vertices. By Proposition 4 the two vertices of the quotient have the same branch-index.

The following proposition lists some properties of quotient maps.

Proposition 5. Let $\mathbf{M}$ be an Archimedean map on an orientable surface of genus $g \geq 1$ and let $\overline{\mathbf{M}}=\mathbf{M} / \operatorname{Aut}^{+}(\mathbf{M})$ be its quotient of valency $\ell$ on an orbifold $\mathcal{O}\left(\gamma ; m_{0}, m_{1}, \ldots, m_{n}\right)$ or $\mathcal{O}\left(\gamma ; m_{0}^{2}, m_{1}, \ldots, m_{n}\right)$, respectively. Then

(1) $3 \leq \ell m_{0} \leq 3+\sqrt{12 g-3}$, 
(2) $m_{i} f_{i}>2$ for every face $\bar{F}_{i}$, where $f_{i}=\left|\bar{F}_{i}\right|$ is the face-valency, $i \in$ $\{1, \ldots, n\}$ and $m_{i}$ is an index of a branch-point associated with $\bar{F}_{i}$,

(3) $\left|\operatorname{Aut}^{+}(\mathbf{M})\right|>\ell m_{0}^{2}$ if $\mathbf{M}$ is of type I,

(4) $\left|\operatorname{Aut}^{+}(\mathbf{M})\right|>\frac{1}{2} \ell m_{0}^{2}$ if $\mathbf{M}$ is of type II.

Proof. Observe that $m_{0}$ is the order of a vertex stabiliser in $\mathbf{M}$ and $k=\ell m_{0}$ is the valency of $\mathbf{M}$. In the case $\ell m_{0} \leq 2$ the lifted map cannot be polyhedral, since its valency is $k \leq 2$.

Now we shall prove the upper bound $k \leq 3+\sqrt{12 g-3}$. Let $v, e$ and $f$ denote the number of vertices, edges and faces of $\mathbf{M}$, respectively. By the Euler-Poincaré equation it holds that $v-e+f=\chi=2-2 g$. By vertex-transitivity, the valency $k$ of a lifted map $\mathbf{M}$ is equal to $k=\ell m_{0}$ and $e=k v / 2$. Since $\mathbf{M}$ has to be simple, $f \leq k v / 3$. If $g=1$, then direct computation yields $k \leq 6$. If $g \geq 2$, then for $k \leq 6$ the upper bound is satisfied. Hence we may assume $k \geq 7$. Inserting the previous terms in the Euler-Poincaré equation and using $v>k$ we get

$$
-\chi \geq k v\left(\frac{1}{6}-\frac{1}{k}\right)>k^{2}\left(\frac{1}{6}-\frac{1}{k}\right) .
$$

Hence $0>k^{2}-6 k+6(-\chi)$ and statement (1) holds.

To see (2) observe that $m_{i} f_{i}$ for $i \geq 1$ is the valency of a face $F_{i}$ covering $\bar{F}_{i}$. Since the face-valency in a polyhedral map is at least 3 we are done.

Denote $\operatorname{Aut}^{+}(\mathbf{M})=\mathrm{G}$. To prove (3) recall that $k=\ell m_{0}$ is the valency of $\mathbf{M}$ while $\frac{|\mathrm{G}|}{\left|\mathrm{G}_{v}\right|}=\frac{|\mathrm{G}|}{m_{0}}$, where $\mathrm{G}_{v}$ is the vertex stabilizer in $\mathrm{G}$. Since $\mathbf{M}$ is a simple map, the number of vertices of $\mathbf{M}$ is $\frac{\left\lfloor\mathrm{Aut}^{+}(\mathbf{M}) \mid\right.}{m_{0}}>\ell m_{0}$.

To see (4) observe that if $\mathbf{M}$ is of type II we have $\frac{v}{2}=\frac{|\mathrm{G}|}{\left|\mathbf{G}_{v}\right|}=\frac{|\mathrm{G}|}{m_{0}}$. Since $\mathbf{M}$ is simple, $v>k=\ell m_{0}$ and consequently $\frac{2|\mathrm{G}|}{m_{0}}>\ell m_{0}$.

\section{Reconstruction of Archimedean maps}

An explicit construction of the lift over $\overline{\mathbf{M}}$ requires a practical method of description of the covering. Such a method is provided by voltage assignments 14. Usually, voltages are assigned to darts. However, in order to encompass all regular covers (including those which are not valency preserving), we need to employ voltage assignments on angles. We shall follow the approach developed in [22].

Let $\mathbf{M}=(D ; R, L)$ be a map. An (oriented) angle of $\mathbf{M}$ is an ordered pair $a=(x, y)$, where $x$ and $y$ are darts of $\mathbf{M}$ such that $y \in\left\{x R, x R^{-1}, x L\right\}$. We always consider the angles $(x, x R)$ and $\left(x, x R^{-1}\right)$ to be distinct. The darts $x$ and $y$ are called the initial and the terminal dart of $a$, respectively. The angle $a^{-1}=(y, x)$ is the inverse of $a=(x, y)$. We denote by $A(\mathbf{M})$ the set of all angles of $\mathbf{M}$; obviously, $|A(\mathbf{M})|=3|D|$.

An angle-walk (or briefly a walk) is a sequence $W=a_{1} a_{2} \ldots a_{n}$ of angles of $\mathbf{M}$ such that the terminal dart of $a_{i}$ coincides with the initial dart of $a_{i+1}$, for each index $i=1,2, \ldots, n-1$. The initial dart of $a_{1}$ and the terminal dart of $a_{n}$ are called the initial and the terminal dart of $W$, respectively. The term closed angle-walk has the obvious meaning. If $W=a_{1} a_{2} \ldots a_{n}$ is a walk originating at $x$ and terminating at $y$, then $W^{-1}=a_{n}^{-1} a_{n-1}^{-1} \ldots a_{1}^{-1}$ is a walk originating at $y$ and terminating at $x$, called the inverse of $W$.

Let $\mathbf{M}$ be a map and let $\mathrm{G}$ be a finite group. A voltage assignment on $\mathbf{M}$ valued in $\mathrm{G}$ is a function $\xi: A(\mathbf{M}) \rightarrow \mathrm{G}$ such that for any angle $a$ one has $\xi\left(a^{-1}\right)=$ 
$\xi(a)^{-1}$. Note that if $x$ is the unique dart of a semiedge, then the voltage $\xi(x, x L)$ is necessarily an involution. In what follows we shall denote $\xi(a)=\xi_{a}$. The group $\mathrm{G}$ is called the voltage group.

A voltage assignment can be extended to walks in the obvious way. Let $W=$ $a_{1} a_{2} \ldots a_{n}$ be an angle-walk on $\mathbf{M}$. The voltage of $W$ is defined to be the product $\xi_{W}=\xi_{a_{1}} \xi_{a_{2}} \ldots \xi_{a_{n}}$. The subgroup of $\mathrm{G}$ generated by voltages of all closed walks based at a fixed dart $x$ will be called the local voltage group $\mathrm{G}^{x}$ at $x$.

Given a voltage assignment $\xi$ on $\mathbf{M}=(D ; R, L)$ valued in $\mathrm{G}$, set $D^{\xi}=D \times \mathrm{G}$, and define the permutations of $D^{\xi}$ by

$$
\begin{aligned}
& (x, h) R^{\xi}=(x R, h \cdot \xi(x, x R)), \\
& (x, h) L^{\xi}=(x L, h \cdot \xi(x, x L)) .
\end{aligned}
$$

If the group $\left\langle R^{\xi}, L^{\xi}\right\rangle$ is transitive, then $\mathbf{M}^{\xi}=\left(D^{\xi} ; R^{\xi}, L^{\xi}\right)$ is the derived map determined by $\mathbf{M}$ and $\xi$. The group $\left\langle R^{\xi}, L^{\xi}\right\rangle$ is transitive if $G^{x}=G$. In what follows we assume this property to be satisfied automatically.

It is easy to see that the natural projection $\pi_{\xi}: \mathbf{M}^{\xi} \rightarrow \mathbf{M}$ erasing the second coordinate is a map covering. Observe that for each element $a \in \mathrm{G}$ the mapping $\psi_{a}:(x, g) \mapsto(x, a g)$ is a fibre-preserving automorphism of $\mathbf{M}^{\xi}$ and that the group $\tilde{\mathrm{G}}=\left\{\psi_{a} ; a \in \mathrm{G}\right\}$ is isomorphic to $G$. Moreover, the projection $\pi_{\tilde{G}}: M^{\xi} \rightarrow \mathbf{M}^{\xi} / \tilde{\mathrm{G}}$ is clearly equivalent to $\pi_{\xi}$. Therefore, $\pi_{\xi}$ is a regular map homomorphism. The converse holds as well; see [22, Theorem 5.1].

Theorem 6 ([22]). Let $\varphi: \tilde{\mathbf{M}} \rightarrow \mathbf{M}=\tilde{\mathbf{M}} / \mathrm{G}$ be a standard map homomorphism. Then there exists a voltage assignment $\xi$ on $\mathbf{M}$ valued in $\mathrm{G}$ such that the natural projection $\pi_{\xi}: \mathbf{M}^{\xi} \rightarrow \mathbf{M}$ is equivalent to $\varphi$.

Let $v$ be a vertex of valency $k$. A boundary angle walk for a vertex $v$ is a closed walk $a_{1} a_{2} \ldots a_{k}$ such that $a_{i}=\left(x R^{i-1}, x R^{i}\right)$ for some dart $x$ emanating from $v, i=1, \ldots, k$. A boundary angle walk for a semiedge $s=\{x\}$ is the closed walk $(x, x L)$. A boundary angle walk for an edge $e=\{x, x L\}, x \neq x L$ is the closed walk $(x, x L)(x L, x)$. A boundary angle walk for a face $F$ of valency $k$ is a closed walk $a_{1} a_{2} \ldots a_{2 k}$ where $a_{2 i}=\left(x(R L)^{i-1} R, x(R L)^{2 i}\right)$ and $a_{2 i-1}=$ $\left(x(R L)^{i-1}, x(R L)^{i-1} R\right), i=1,2, \ldots, k$. For $y \in V \cup E \cup F$ we set $\xi_{y}=\xi_{W}$, where $W$ is the boundary angle walk for $y$. Let $\psi$ be a map automorphism on $\mathbf{M}$. We say that a voltage-assignment $\xi$ on $\mathbf{M}$ is locally- $\psi$-invariant if for every closed angle walk $W$ we have $\xi_{W}=1$ implies $\xi_{\psi W}=1$ [22, p. 455].

Let $\mathbf{M}$ be a map on an orbifold $\mathcal{O}\left(\gamma ; m_{1}, m_{2}, \ldots, m_{r}\right)$ with a distribution function $b: V \cup E \cup F \rightarrow\left\{1, m_{1}, m_{2}, \ldots, m_{r}\right\}$. A voltage-assignment $\xi$ on $\mathbf{M}$ will be called $b$-compatible if for every $y \in V \cup E \cup F$ we have $\left|\xi_{y}\right|=b(y)$.

The next theorem follows from Theorem 6 and Propositions 3 and 4

Theorem 7. Let $\varphi: \mathbf{M} \rightarrow \overline{\mathbf{M}}=\mathbf{M} / \operatorname{Aut}^{+}(\mathbf{M})$ be a regular covering from an Archimedean map of genus $g$ and let $b$ be the induced distribution of branch-indices. Then there exists a b-compatible voltage-assignment $\xi: A(\overline{\mathbf{M}}) \rightarrow \operatorname{Aut}^{+}(\mathbf{M})$ such that the natural projection $\pi_{\xi}: \mathbf{M}^{\xi} \rightarrow \overline{\mathbf{M}}$ is equivalent to $\varphi$.

Furthermore, if $\mathbf{M}$ is of type II, then $\xi$ is locally- $\bar{\tau}$-invariant for some orientationreversing map automorphism $\bar{\tau}$ transposing the two vertices of $\overline{\mathbf{M}}$.

Remark 8. Let $\mathbf{N}$ be a map. Using some standard arguments in topological graph theory [14, Theorem 2.5.3, Theorem 2.5.4] one can prove that a voltage assignment $\xi: A(\mathbf{N}) \rightarrow \mathrm{G}$ can be reduced with respect to a rooted spanning tree $T$ of $\mathbf{N}$ as 
follows. For each vertex $v$ there is a unique dart $d_{v}$ (based at $v$ ) on the unique path in $T$ joining $v$ to the root. We form an equivalent voltage assignment satisfying the following conditions:

(1) all angles of the form $(x, x R),\left(x, x R^{-1}\right)$ except $\left(d_{v}, d_{v} R\right),\left(d_{v} R, d_{v}\right), v \in$ $V(\mathbf{N})$, receive trivial voltages,

(2) for angles of the form $a=(x, x L), \xi_{a}=1$ if $x$ is contained in the spanning tree $T$.

The assignment $\xi$ satisfying (1) and (2) will be called $T$-reduced. In a $T$-reduced voltage assignment $\xi$, the voltage $\xi_{a}$ on an angle $a=(x, x L)$ can be interpreted as a voltage on the dart $x$ while for every vertex $v \in V$ the voltage on the angle $\left(d_{v}, d_{v} R\right)$ can be interpreted as $\xi_{v}$. It follows that a $T$-reduced voltage assignment can be viewed as a voltage assignment $\xi: V \cup D \rightarrow \mathrm{G}$.

Remark 9. A $b$-compatible voltage assignment $\xi: A(\overline{\mathbf{M}}) \rightarrow \mathrm{G}$ on a one-vertex map $\overline{\mathbf{M}}$ on an orbifold $\mathcal{O}=\mathcal{O}\left(\gamma ; m_{1}, m_{2}, \ldots, m_{r}\right)$ determines $\mathrm{G}$ as a quotient of the fundamental group $\pi_{1}(\mathcal{O})$ of the orbifold $\mathcal{O}$ which is an F-group with presentation:

$$
\pi_{1}(\mathcal{O})=\left\langle a_{1}, \ldots, a_{\gamma}, b_{1}, \ldots, b_{\gamma}, x_{1}, \ldots, x_{r} \mid x_{1}^{m_{1}}, \ldots, x_{r}^{m_{r}}, \prod_{j=1}^{r} x_{j} \prod_{k=1}^{\gamma}\left[a_{k}, b_{k}\right]\right\rangle .
$$

Given an orbifold $\mathcal{O}$ the canonical one-vertex map is the one whose faces induce the universal relators in the above presentation [12, Section 1.2].

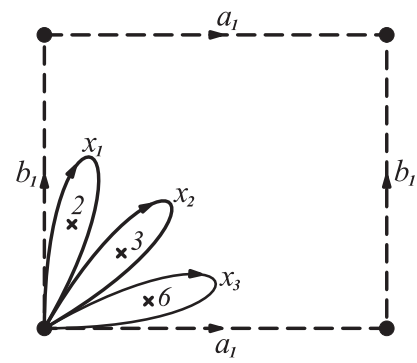

Figure 1. The canonical map on the orbifold $\mathcal{O}(1 ;\{2,3,6\})$ defines F-group $\pi(\mathcal{O})=\left\langle a_{1}, b_{1}, x_{1}, x_{2}, x_{3} \mid x_{1}^{2}, x_{2}^{3}, x_{3}^{6}, x_{1} x_{2} x_{3}\left[a_{1}, b_{1}\right]\right\rangle$.

\section{Description of COMputation}

At this moment we are ready to determine Archimedean maps of a given genus. In what follows we provide steps of an algorithm reconstructing all Archimedean maps of genera $g=2,3,4$.

Step 1 (Solving Riemann-Hurwitz equation). The lists of admissible orbifolds were derived in [5] for genus 2 and 3 and in [2] for surface of genus 4 . For our purpose we have derived the catalogues independently. It turned out that there is a misprint in the catalogue in [2] (compare with [19]). The processing is divided into two substeps:

a) solving the Riemann-Hurwitz equation numerically, 
b) for a given set of parameters $|\mathrm{G}|, g, \gamma, m_{1}, m_{2}, \ldots, m_{r}$ we determine all groups $\mathrm{G}$ acting on $\mathcal{S}_{g}$ giving rise to the $g$-admissible orbifold $\mathcal{O}=$ $\mathcal{O}\left(\gamma ; m_{1}, m_{2}, \ldots, m_{r}\right)$. This can be done by constructing all $b$-compatible voltage assignments defined on a canonical one-vertex map on $\mathcal{O}$.

In the first step the following bound turned out to be useful.

Lemma 10. Given $g>1$, the number of branch-indices $r$ in the Riemann-Hurwitz equation (2.1) is bounded by $2 g+2 \geq r$.

Proof. Assume the covering is branched. Hence $|\mathrm{G}| \geq 2$. Setting $m_{i}=2$ for each $i=1,2, \ldots, r$, minimises the summands and maximises $r$. Now

$$
2-2 \gamma+\frac{2 g-2}{|\mathrm{G}|}=\frac{r}{2} .
$$

To maximise $r$ we set $\gamma=0$ and $|\mathrm{G}|=2$.

The computation iterates through $\gamma \in\{0 \ldots g-1\}$ and $|\mathrm{G}| \in\{2, \ldots, 84(g-1)\}$ testing the values of $m_{i}$ such that $m_{i} \leq|\mathrm{G}|$ divides $|\mathrm{G}|$ for each $i=1,2, \ldots, r$ including cases with $r=0$ (smooth covers $\mathcal{S}_{g} \rightarrow \mathcal{S}_{\gamma}$ ).

Note that all the groups of orders given by the numerical solution are contained (at least up to genus 15, see [19]) in Small Groups Library [29], which is contained in Magma [3. Thus employing the software Magma, the candidates for the voltage groups can be identified inside these lists.

Step 2 (Determination of quotient maps). Given a group $\mathrm{G} \leq \mathrm{Aut}^{+}(\mathbf{M})$ acting with one or two orbits on the set of vertices of $\mathbf{M}$, we use Proposition 5 to obtain the maximal valency $\ell$ of the quotient map $\overline{\mathbf{M}}=(\bar{D} ; \bar{R}, \bar{L})$. In the one-vertex case we assume $\bar{R}=(1,2, \ldots, \ell)$ and in the two-vertex case we assume $\bar{R}=(1,2, \ldots, \ell)(\ell+$ $1, \ell+2, \ldots, 2 \ell)$. For sake of clarity the two cycles of $\bar{R}$ in two-vertex quotient map are denoted as the "black" and "white" vertex. Every quotient map is determined by setting $\bar{L} \in \operatorname{Sym}(\ell)$ or $\bar{L} \in \operatorname{Sym}(2 \ell)$, respectively, where $\bar{L}^{2}=1$ and $\langle\bar{R}, \bar{L}\rangle$ is transitive on $\bar{D}$. In the case of a two-vertex quotient we first determine all $\bar{\tau}$ transposing the two vertices such that $\bar{R}^{\bar{\tau}}=\bar{R}^{-1}$ (see Proposition 4) and then we determine all dart-reversing involutions $\bar{L}$ such that $\bar{L}^{\bar{\tau}}=\bar{L}$. This approach significantly decreases the computation time. In the case of a one-vertex quotient map $\bar{\tau}$ is always set to be the identity of $\operatorname{Sym}(\ell)$. By reasons of symmetry it is sufficient to consider a unique representative of each conjugacy class $\bar{L}\langle\bar{R}\rangle$.

We fix one dart $d$ of $\overline{\mathbf{M}}$ to be a root and reorder the branch-indices such that the face whose boundary walk contains $d$ has branch-index $m_{1}$. In the one-vertex case we simply choose the dart $d=1$. In the two-vertex case we choose a dart emanating from the "white" vertex and terminating in the "black" vertex.

Given a quotient map $\overline{\mathbf{M}}$ of genus $\gamma$ and a $g$-admissible orbifold $\mathcal{O}\left(\gamma ; m_{1}\right.$, $\left.m_{2}, \ldots, m_{r}\right)$, we derive all possible distributions of branch-points among the faces, semiedges and vertices of $\overline{\mathbf{M}}$. The distribution $b$ of branch-indices is done such that the conditions of Proposition 5 are satisfied. The distribution $b$ is checked for consistency with $\bar{\tau}$, i.e., the images of vertices, semiedges and faces should have the same branch-assignment as the respective preimages. The search of possible quotient embeddings is done through all $g$-admissible signatures (from Step 1) and all quotient maps. 

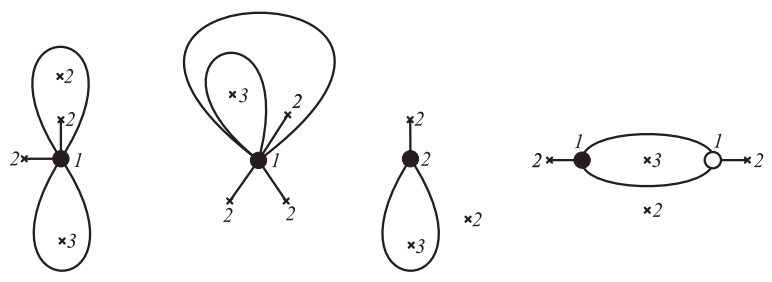

Figure 2. Different quotients on the orbifold $\mathcal{O}(0 ;\{2,2,2,3\})$.

Step 3 (Determining voltage assignments). By an embedding $\overline{\mathbf{M}} \hookrightarrow \mathcal{O}$ a partial presentation of a voltage group is determined. Namely, for each angle $\bar{a}$ we have a generator $\xi_{\bar{a}}$ and each $x \in \bar{V} \cup \bar{E} \cup \bar{F}$ gives rise to the relator $\xi_{x}^{b(x)}$, where $\xi_{x}$ is written as a product of generators. This way a universal voltage group $\mathrm{U}$ is defined. Given $\overline{\mathbf{M}}$ on the orbifold $\mathcal{S}_{g} / \mathrm{G}$ we try to construct an order-preserving epimorphism $\xi$ : $\mathrm{U} \rightarrow \mathrm{G}$. Actually, we use Magma HomomorphismsProcess procedure, as described in The Magma Handbook of Functions [6, Part IX].

In fact, following Remark 8 we are going to construct a $T$-reduced $b$-compatible voltage assignment $\xi: \bar{V} \cup \bar{D} \rightarrow \mathrm{G}$, which allows us to reduce the number of generators. Keeping in mind that the derived map has to be simple we get additional constrains:

- for every dart $x$ in $\bar{D}$ not belonging to $T, \xi_{x} \neq 1$,

- two darts, $x, y$ emanating from the same vertex and terminating in the same vertex are endowed with different voltages, i.e., $\xi_{x} \neq \xi_{y}$,

Step 4 (Lifting the maps). The construction described in (3.1) was used to determine Archimedean maps. The lifted maps were tested whether they satisfy the required criteria (genus, number of darts, vertices, etc.).

In the case of two-vertex quotient maps we have tested whether the automorphism $\bar{\tau}$ lifts. For this purpose we have recorded the darts in the fibre over a dart emanating from the "black" or from the "white" vertex of the quotient, respectively. We choose a dart $y$ from the "black fibre" covering a fixed dart $d$ in the quotient and determine the set of darts $\left\{x_{1}, x_{2}, \ldots, x_{|G|}\right\}$ from the "white fibre" covering the dart $d \cdot \bar{\tau}$. We check whether at least one mapping $\tau: y \mapsto x_{i}$ extends into a map homomorphism $\tau: \mathbf{M} \rightarrow \mathbf{M}^{-1}$.

Step 5 (Polyhedrality test). After completing Step 4 we have obtained a list of simple maps of a given genus in terms of $\mathbf{M}=(D ; R, L)$. Each $\mathbf{M}$ in the list is vertex-transitive. The polyhedrality is checked directly for each map in the list following the definition.

Step 6 (Recognition of isomorphism classes). The list of polyhedral maps may contain (and actually it contains) isomorphic maps represented differently. In fact, in the previous computations we have constructed all the pairs of the form $(\mathbf{M}, \mathrm{G})$, where $\mathbf{M}$ is an Archimedean map on $\mathcal{S}_{g}$ and $\mathrm{G} \leq \operatorname{Aut}^{+}(\mathbf{M})$ acts either with one, or with two orbits on vertices of $\mathbf{M}$. To solve the isomorphism problem for a pair of maps $\mathbf{M}_{1}$ and $\mathbf{M}_{2}$ the following observation is crucial. Given a mapping $\psi$ of a dart $x \mapsto y, x \in D_{1}$ and $y \in D_{2}$, employing the commuting rules $R_{1}^{\psi}=R_{2}$ and 
$L_{1}^{\psi}=L_{2}$ the mapping $\psi$ either extends to a unique isomorphism $\psi: \mathbf{M}_{1} \rightarrow \mathbf{M}_{2}$, or there is no such isomorphism. The algorithms determining Aut(M), checking whether $\mathbf{M}$ is reflexible or chiral and so on, reduce to the same procedure.

\section{Census of Archimedean maps of genera 2, 3 And 4}

Each map in the list in Appendix $\mathrm{A}$ is described by a three-row record. The first row contains the following description of an Archimedean map

$$
A_{g . i}=\mathbf{M}\left(\mathrm{G}_{g . i}, \mathrm{G}_{v},\left(x_{1}, x_{2}, \ldots, x_{\ell}\right)^{m_{0}}\right),
$$

where $g$ is genus and $i$ is a unique integer identifier. The group $\mathrm{G}=\mathrm{G}_{g . i} \leq$ $\operatorname{Aut}^{+}(\mathbf{M})$ is either vertex-transitive or it acts on the vertex set with two orbits. The group $\mathrm{G}_{v}=\langle z\rangle \leq \mathrm{G}$ is a vertex stabilizer $\left(\mathrm{G}_{v} \cong C_{m_{0}}\right.$, where $\left.m_{0} \mid \operatorname{val}(\mathbf{M})\right)$. In general, the same map admits more than one vertex-transitive action of a group of automorphisms. Our program chooses the representative with the minimum order. The (local) type of $\mathbf{M}$ and information about the type (Type I or II) and reflexibility is displayed in this row.

In the case of maps of Type I the vector $\left(x_{1}, x_{2}, \ldots, x_{\ell}\right)$ gives a fixed cyclic order of the set of generators $\left\{x_{1}, x_{2}, \ldots, x_{\ell}\right\}$ induced by the rotation of $\mathbf{M}$. The set $\left\{x_{1}, x_{2}, \ldots, x_{\ell}\right\}$ is closed under taking inverses. The above description generalises the standard description of a Cayley map 28. A map $\mathbf{M}$ is a Cayley map if there exist $\mathrm{G} \leq \mathrm{Aut}^{+}(\mathbf{M})$ acting regularly on the vertices of $\mathbf{M}$.

In case of maps of Type II the vector $\left(x_{1}, x_{2}, \ldots, x_{\ell}\right)$ gives a fixed cyclic order of the set of generators $\left\{x_{1}, x_{2}, \ldots, x_{\ell}\right\}$ at the "black" vertices induced by the rotation of $\mathbf{M}$. The rotation at the "white" vertices is compatible with the cyclic permutation $\left(\bar{\tau} x_{1}, \bar{\tau} x_{2}, \ldots, \bar{\tau} x_{\ell}\right)$. In the case $m_{0}=1$ the action of the considered automorphism group is regular on vertices and the corresponding Archimedean map is an unoriented Cayley map (see 20, for more details).

The second row of the record shows a presentation of $\mathrm{G}=\mathrm{G}_{g . i}$ and structural information about $\mathrm{G}[3,29$.

The last row gives the quotient map $\overline{\mathbf{M}}=(\bar{D} ; \bar{R}, \bar{L})$; we use the abbreviation $\overline{\mathbf{M}}=[\bar{R} ; \bar{L}]$. If $\mathbf{M}$ is of Type $\mathrm{I}$, then $\bar{R}$ is by definition $\bar{R}=(1,2, \ldots, \ell$ ) (see Theorem (11), and $\bar{L} \in \operatorname{Sym}(\ell)$ is written as a product of transpositions. If $\mathbf{M}$ is of Type II, then $\bar{R}$ is by definition $\bar{R}=(1,2, \ldots, \ell)(\ell+1, \ell+2, \ldots, 2 \ell)$ (see Theorem 11), and $\bar{L} \in \operatorname{Sym}(2 \ell)$ is written as a product of transpositions. The quotient $\bar{M}$ is embedded into the orbifold of the signature $\mathcal{O}\left(\gamma ; m_{1}, m_{2}, \ldots, m_{r}\right)=\mathcal{S}_{g} / \mathrm{G}_{g . i}$; see the extended catalogue [18].

The records in the list are sorted lexicographically according to the local type. Full records of Archimedean maps of genera 2,3,4 can be found at the website [18].

The output of our computation can be found in the following theorems.

Theorem 11. The following table enumerates Archimedean maps of genera 2, 3 and 4 .

\begin{tabular}{|l|c|c|c|c|c|}
\hline Genus & All & Type I & Type II & Reflexible & Chiral pairs \\
\hline 2 & 17 & 9 & 8 & 13 & 4 \\
3 & 103 & 78 & 25 & 63 & 40 \\
4 & 111 & 76 & 35 & 77 & 34 \\
\hline
\end{tabular}


Since our algorithm constructs all pairs $(\mathbf{M}, \mathbf{G})$ where $\mathbf{M}$ is Archimedean of genus $2 \leq g \leq 4$ and $\mathbf{G} \leq \operatorname{Aut}^{+}(\mathbf{M})$ is either transitive on vertices of $\mathbf{M}$ or it acts with two orbits, we get as a by-product a list of non-Cayley Archimedean maps of genera 2,3,4. Most of the Archimedean maps of type I of small genera are Cayley maps.

Theorem 12. Every Archimedean map of type I and of genus two is a Cayley map. There are $\mathbf{3}$ non-Cayley Archimedean maps of genus three of type I. There are $\mathbf{2}$ non-Cayley Archimedean maps of genus four and of type $I$.

The description of non-Cayley Archimedean maps and genus $2 \leq g \leq 4$ follows.

A $3.191 \quad \mathbf{M}\left(\mathrm{G}_{3.191}, C_{3},(x)^{3}\right)$ is of local type $\left(7^{3}\right)$, type I, reflexible; $\mathrm{G}_{3.191}=\left\langle x, y \mid x^{2}, y^{3},(x y)^{7},\left(x y^{-1} x y\right)^{4}\right\rangle \cong P S L_{3}(2) ;$

$\overline{\mathbf{M}}=[(1) ;()]$ on $\mathcal{O}(0 ;\{2,3,7\})$;

$\mathrm{A}_{3.382} \mathbf{M}\left(\mathrm{G}_{3.382}, C_{2},\left(x, x^{-1}\right)^{2}\right)$ is of local type (3.7.3.7), type I, reflexible; $\mathrm{G}_{3.382}=\left\langle x, y \mid y^{2},(x y)^{3}, x^{7},\left(y x^{-3}\right)^{4}\right\rangle \cong P S L_{3}(2)$; $\overline{\mathbf{M}}=[(1,2) ;(1,2)]$ on $\mathcal{O}(0 ;\{2,3,7\})$.

$\mathrm{A}_{3.1383} \mathbf{M}\left(\mathrm{G}_{3.1383}, C_{4},\left(x, x^{-1}\right)^{4}\right)$ is of local type $\left(3^{8}\right)$, type I, reflexible; $\mathrm{G}_{3.1383}=\left\langle x, y \mid x^{3}, y^{4},\left(x^{-1} y^{-1}\right)^{3},\left(y x^{-1}\right)^{3}\right\rangle \cong\left(C_{4} \times C_{4}\right): C_{3}$; $\overline{\mathbf{M}}=[(1,2) ;(1,2)]$ on $\mathcal{O}(0 ;\{3,3,4\})$.

A4.656 $\mathbf{M}\left(\mathrm{G}_{4.656}, C_{2},\left(x, x^{-1}\right)^{2}\right)$ is of local type $\left(5^{4}\right)$, type I, reflexible; $\mathrm{G}_{4.656}=\left\langle x, y \mid y^{2}, x^{5},\left(x^{2} y\right)^{3},\left(y x y x^{-2}\right)^{2},(x y)^{5}\right\rangle \cong A_{5}$; $\overline{\mathbf{M}}=[(1,2) ;(1,2)]$ on $\mathcal{O}(0 ;\{2,4,5\})$.

A4.1874 $\mathbf{M}\left(\mathrm{G}_{4.1874}, C_{2},\left(z, z^{-1}, x, y\right)^{2}\right)$ is of local type $\left(3^{3} .4 .3^{3} .4\right)$, type I, reflexible; $\mathrm{G}_{4.1874}=\left\langle x, y, z, u \mid u^{2}, x^{2}, y^{2}, z^{3}, z^{-1} x y, z x z^{-1} y,(z u y)^{2},(u y)^{3},(u x)^{3},(y x u x)^{2}\right\rangle$ $\cong S_{4}$

$\overline{\mathbf{M}}=[(1,2,3,4) ;(1,2)]$ on $\mathcal{O}(0 ;\{2,2,2,4\})$.

\section{Appendix A. Archimedean maps of genus 2}

In what follows, we display the record containing basic data for Archimedean maps of genus two only. For genus 3 and 4 the list with other additional information can be found at the website [18].

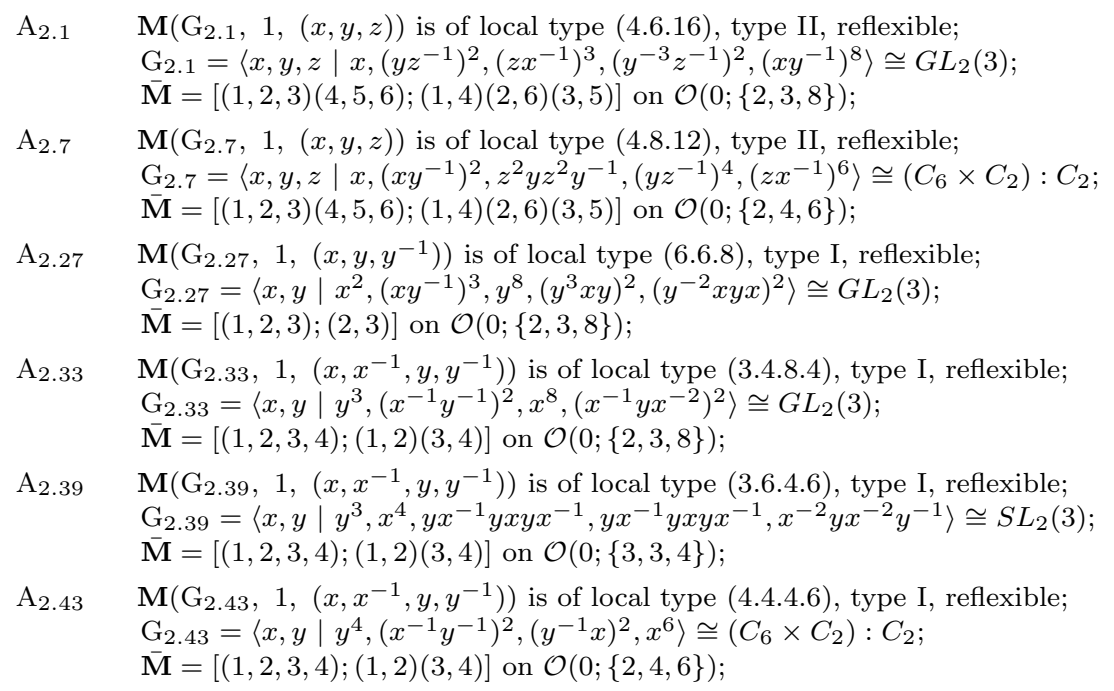


$\mathrm{A}_{2.46} \mathbf{M}\left(\mathrm{G}_{2.46}, 1,(z, r, x, s)\right)$ is of local type (4.4.4.6), type II, reflexible;

$\mathrm{G}_{2.46}=\left\langle x, y, z, r, s \mid z, x^{2}, y^{2}, s^{2},(s x)^{2}, r s x y, r^{-1} s r s,(y s)^{2},\left(s z^{-1}\right)^{2},\left(z r^{-1}\right)^{3}\right\rangle$ $\cong D_{12}$

$\overline{\mathbf{M}}=[(1,2,3,4)(5,6,7,8) ;(1,5)(2,8)(4,6)]$ on $\mathcal{O}(0 ;\{2,2,2,3\}) ;$

$\mathrm{A}_{2.51} \quad \mathbf{M}\left(\mathrm{G}_{2.51}, 1,(x, y, z, r)\right)$ is of local type (4.4.4.6), type II, reflexible; $\mathrm{G}_{2.51}=\left\langle x, y, z, r \mid x, z^{2},(y z)^{2},\left(r x^{-1}\right)^{2},\left(z r^{-1}\right)^{2},\left(r^{-1}, y^{-1}\right),\left(x y^{-1}\right)^{3}\right\rangle \cong D_{12}$ $\overline{\mathbf{M}}=[(1,2,3,4)(5,6,7,8) ;(1,5)(2,8)(3,7)(4,6)]$ on $\mathcal{O}(0 ;\{2,2,2,3\})$;

$\mathrm{A}_{2.89} \quad \mathbf{M}\left(\mathrm{G}_{2.89}, 1,\left(x, y, y^{-1}, z, z^{-1}\right)\right)$ is of local type $\left(3^{4} .8\right)$, type I, chiral; $\mathrm{G}_{2.89}=\left\langle x, y, z \mid x^{2}, x y^{-1} z^{-1}, z^{3}, y^{8}, z y^{-2} x z^{-1} y^{-3}\right\rangle \cong G L_{2}(3) ;$

$\overline{\mathbf{M}}=[(1,2,3,4,5) ;(2,3)(4,5)]$ on $\mathcal{O}(0 ;\{2,3,8\})$;

A 2.93 $\quad \mathbf{M}\left(\mathrm{G}_{2.93}, 1,\left(x, y, y^{-1}, z, z^{-1}\right)\right)$ is of local type (3.3.4.3.6), type I, chiral; $\mathrm{G}_{2.93}=\left\langle x, y, z \mid x^{2}, x y^{-1} z^{-1}, y^{4},\left(z^{-1} y\right)^{2}, z^{6}\right\rangle \cong\left(C_{6} \times C_{2}\right): C_{2}$; $\overline{\mathbf{M}}=[(1,2,3,4,5) ;(2,3)(4,5)]$ on $\mathcal{O}(0 ;\{2,4,6\})$;

$\mathrm{A}_{2.97} \quad \mathbf{M}\left(\mathrm{G}_{2.97}, 1,\left(z, r, s, s^{-1}, x\right)\right)$ is of local type (3.4 $)$, type II, reflexible; $\mathrm{G}_{2.97}=\left\langle x, y, z, r, s, t \mid z, x^{2}, y^{2}, y x, s^{-1} t^{-1}, s^{3}, t^{3}, r x s^{-1}, r s^{-1} x z^{-1}, z r^{-1} y t^{-1}\right\rangle$ $\cong C_{6}$

$\overline{\mathbf{M}}=[(1,2,3,4,5)(6,7,8,9,10) ;(1,10)(2,6)(3,4)(8,9)]$ on $\mathcal{O}(0 ;\{2,2,3,3\}) ;$

$\mathrm{A}_{2.99} \quad \mathbf{M}\left(\mathrm{G}_{2.99}, 1,\left(x, x^{-1}, y, y^{-1}, z, z^{-1}\right)\right)$ is of local type $\left(3^{5} .4\right)$, type I, reflexible; $\mathrm{G}_{2.99}=\left\langle x, y, z \mid x^{3}, z^{3}, x^{-1} y^{-1} z^{-1}, y^{4}, x y^{-2} z y^{-1}\right\rangle \cong S L_{2}(3) ;$ $\overline{\mathbf{M}}=[(1,2,3,4,5,6) ;(1,2)(3,4)(5,6)]$ on $\mathcal{O}(0 ;\{3,3,4\})$

$\mathrm{A}_{2.105} \mathbf{M}\left(\mathrm{G}_{2.105}, 1,\left(x, z, z^{-1}, r, y, r^{-1}\right)\right)$ is of local type $\left(3^{4} .4^{2}\right)$, type I, chiral; $\mathrm{G}_{2.105}=\left\langle x, y, z, r \mid x^{2}, y^{2}, x z^{-1} r^{-1}, z^{3}, z r^{-2}, x z r,(r y)^{2},(y x)^{2},\left(z^{-1} y\right)^{2}\right\rangle \cong D_{12} ;$ $\overline{\mathbf{M}}=[(1,2,3,4,5,6) ;(2,3)(4,6)]$ on $\mathcal{O}(0 ;\{2,2,2,3\})$;

A $2.109 \quad \mathbf{M}\left(\mathrm{G}_{2.109}, 1,\left(z, z^{-1}, r, s, r^{-1}, x\right)\right)$ is of local type $\left(3^{4} \cdot 4^{2}\right)$, type II, reflexible; $\mathrm{G}_{2.109}=\langle x, y, z, r, s, t, u| s, x^{2}, y^{2}, y x, t z^{-1}, z u r^{-1}$, $\left.t^{3}, z^{3}, z^{-1} r^{-1} x, x z u^{-1}, t y u^{-1}, r s^{-1} u s\right\rangle \cong C_{6} ;$

$\overline{\mathbf{M}}=[(1,2,3,4,5,6)(7,8,9,10,11,12) ;(1,2)(3,5)(4,10)(7,12)(9,11)]$ on $\mathcal{O}(0 ;\{2,2,3,3\}) ;$

$\mathrm{A}_{2.111} \mathbf{M}\left(\mathrm{G}_{2.111}, 1,\left(r, r^{-1}, s, x, y, s^{-1}, z\right)\right)$ is of local type $\left(3^{7}\right)$, type I, chiral; $\mathrm{G}_{2.111}=\left\langle x, y, z, r, s \mid x^{2}, y^{2}, z^{2}, r s^{-2}, r^{3}, r^{-1} s^{-1} z, s x y, z r s,(z x)^{2}\right\rangle \cong D_{12}$; $\overline{\mathbf{M}}=[(1,2,3,4,5,6,7) ;(1,2)(3,6)]$ on $\mathcal{O}(0 ;\{2,2,2,3\})$;

$\mathrm{A}_{2.114} \mathbf{M}\left(\mathrm{G}_{2.114}, 1,\left(z, z^{-1}, r, s, t, r^{-1}, x\right)\right)$ is of local type $\left(3^{7}\right)$, type II, reflexible; $\mathrm{G}_{2.114}=\langle x, y, z, v, r, s, t, u| s, x^{2}, y^{2}, y x, u z^{-1}, r s^{-1} t$, $\left.s t^{-1} v, z^{3}, z^{-1} r^{-1} x, u y v^{-1}, u^{3}, z t r^{-1}, x z t^{-1}\right\rangle \cong C_{6} ;$

$\overline{\mathbf{M}}=[(1,2,3,4,5,6,7)(8,9,10,11,12,13,14) ;(1,2)(3,6)(4,11)(5,12)(8,14)(10,13)]$ on $\mathcal{O}(0 ;\{2,2,3,3\})$;

$\mathrm{A}_{2.116} \mathbf{M}\left(\mathrm{G}_{2.116}, 1,\left(z, r, x, s, t, t^{-1}, u\right)\right)$ is of local type $\left(3^{7}\right)$, type II, reflexible; $\mathrm{G}_{2.116}=\langle x, y, z, v, r, s, t, u| z, x^{2}, y^{2}, x y, t r^{-1}, t^{-1} v^{-1}$ $\left.u z^{-1} y, t^{3}, s t^{-1} u^{-1}, v^{3}, r y s^{-1}, z r^{-1} v^{-1}\right\rangle \cong C_{6}$

$\overline{\mathbf{M}}=[(1,2,3,4,5,6,7)(8,9,10,11,12,13,14) ;(1,12)(2,9)(4,8)(5,6)(7,14)(10,11)]$ on $\mathcal{O}(0 ;\{2,2,3,3\})$;

\section{ACKNOWLEDGEMENTS}

The authors would like to thank Jin-Ho Kwak, Marston Conder, and Daniel Pellicer for fruitful discussions about the method and algorithms used here.

\section{REFERENCES}

[1] L. Babai, Vertex-transitive graphs and vertex-transitive maps, J. Graph Theory 15 (1991), no. 6, pp. 587-627. MR1133814 (92m:05064)

[2] O. V. Bogopolski, Classifying the actions of finite groups on orientable surfaces of genus 4, Siberian Adv. Math. 7 (1997), pp. 9-38. MR1604157 (2000e:30083)

[3] W. Bosma, J. Cannon and C. Playoust, The Magma algebra system. I. The user language, J. Symbolic Comput., 24(3-4):235-265, 1997. MR.1484478 
[4] U. Brehm and E. Schulte, Polyhedral maps in "Handbook of discrete and computational geometry", J. E. Goodman and J. O'Rourke (Eds.), Chapman \& Hall/CRC, Boca Raton, FL, 2004, pp. 477-491. MR1730174

[5] S. A. Broughton, Classifying finite group actions on surfaces of low genus, J. Pure Appl. Algebra 69 (1991), pp. 233-270. MR.1090743 (92b:57021)

[6] J. Cannon, W. Bosma, C. Fieker and A. Steel (Eds.), Handbook of Magma Functions, Edition 2.16 (2010), Sydney, 5017 p.

[7] M. Conder and P. Dobcsányi, Determination of all regular maps of small genus, J. Combin. Theory Ser. B 81 (2001), pp. 224-242. MR.1814906 (2002f:05088)

[8] J. H. Conway, H. Burgiel and Ch. Goodman-Strass, "Symmetries of things", A. K. Peters Ltd. 2008. MR2410150 (2009c:00002)

[9] H. M. Cundy and A. P. Rollett, "Mathematical models", 2nd Ed. Oxford University Press, 1961. MR0124167 (23:A1484)

[10] J. D. Dixon and B. Mortimer, "Permutation groups", Springer-Verlag, New York, 1996. MR:1409812 (98m:20003)

[11] The GAP Group, GAP - Groups, Algorithms, and Programming, Version 4.4; 2006, (http://www.gap-system.org).

[12] G. Gromadzki, "Groups of Automorphisms of Compact Riemann and Klein Surfaces", Wydawnictwo U. W. Sz. Pedag., Bydgoszc, 1993.

[13] A. Hurwitz, Über algebraische Gebilde mit Eindeutigen Transformationen in sich, Mathematische Annalen 41 (3), (1893), pp. 403-442 MR.1510753

[14] J. L. Gross and T. W. Tucker, "Topological graph theory", Dover Publications Inc., Mineola, NY, 2001, second edition. MR.1855951

[15] B. Grünbaum, "Convex polytopes", Graduate Texts in Mathematics 221, Springer-Verlag, New York, 2003, second edition. MR1976856 (2004b:52001)

[16] B. Grünbaum and G. C. Shephard, "Tilings and Patterns", W. H. Freeman and Co., New York, 1986. MR.992195(90a:52027)

[17] G. A. Jones and D. Singerman, Theory of maps on orientable surfaces, Proc. London Math. Soc. (3) 37 (1978), pp. 273-307. MR0505721 (58:21744)

[18] J. Karabáš, Archimedean solids, http://www.savbb.sk/ karabas/science.html\#arch.

[19] J. Karabáš, Actions of finite groups on Riemann surfaces of higher genera, http://www.savbb.sk/ karabas/science.html\#rhsu.

[20] J. H. Kwak and Y. S. Kwon, Unoriented Cayley Maps, Studia Scientiarum Mathematicarum Hungarica 43(2) (2006), pp. 137-157 MR2229619 (2007g:05049)

[21] A. Malnič, R. Nedela and M. Škoviera, Lifting graph automorphisms by voltage assignments, European J. Combin. 21 (2000), no. 7, pp. 927-947. MR.1787907 (2001i:05086)

[22] A. Malnič, R. Nedela and M. Škoviera, Regular homomorphisms and regular maps, European J. Combin. 23 (2002), no. 4, pp. 449-461. MR.1914482 (2003g:05045)

[23] A. Mednykh and R. Nedela, Enumeration of unrooted maps of a given genus, J. Combin. Theory Ser. B 96 (2006), pp. 706-729. MR2236507 (2007g:05088)

[24] B. Mohar and C. Thomassen, "Graphs on Surfaces", John Hopkins University Press, Baltimore, 2001. MR 1844449 (2002e:05050)

[25] R. Nedela and M. Skoviera, Exponents of orientable maps, Proc. London Math. Soc. (3), 75 (1997), no. 1, pp. 1-31. MR1444311 (98i:05059)

[26] D. Pellicer and A. Ivić Weiss, Uniform maps on surfaces of non-negative Euler characteristic, preprint.

[27] V. Proulx, "Classification of the Toroidal Groups", Ph.D. thesis, Columbia University, 1977. MR2627033

[28] R. B. Richter, J. Širáň, R. Jajcay, T. W. Tucker and M. E. Watkins, Cayley maps, J. Combin. Theory Ser. B 95 (2005), pp. 189-245. MR2171363 (2006g:05063)

[29] "The Small Groups Library", http://www-public.tu-bs.de:8080/ hubesche/small.html.

[30] E. Steinitz, Polyeder und raumeinteilungen, "Enzyklopedie Math. Wiss". 3 (Geometrie), Leipzig, 1922, pp. 1 - 139.

[31] O. Šuch, Vertex-transitive maps on torus, preprint.

[32] "Archimedean solid", Wikipedia, http://en.wikipedia.org/wiki/Archimedean_solid. 
(J. Karabáš) Science and Research Institute, Matej Bel University, Ďumbierska 1, 97411 Banská Bystrica, Slovakia

E-mail address: karabas@savbb.sk

(R. Nedela) Faculty of Natural Sciences, Matej Bel University, Tajovského 40, 97401 Banská Bystrica, Slovakia

(R.Nedela) Mathematical Institute, Slovak Academy of Sciences, Ďumbierska 1, 97411 Banská Bystrica, Slovakia

E-mail address: nedela@savbb.sk 